\title{
AHA/ACC/ESC 2006 atrial fibrillation guidelines: looking towards the future
}

\author{
Bernard J Gersh and Douglas Packer
}

An old proverb states that "if it looks like a duck, quacks like a duck, and swims like a duck - then it probably is a duck". Nonetheless, there are many species of duck, and they come in different sizes and colors, and occupy various habitats. So is it the case with atrial fibrillation. Although this condition is a single electrocardiographic entity, there is marked diversity in its clinical presentations, symptoms, and prognosis. In addition, the range of therapeutic options available is wide-rate versus rhythm control, pharmacologic and nonpharmacologic treatments, and differing approaches to anticoagulation. Moreover, treatment decisions can be heavily influenced by the nature of the medical center to which the patient presents, the facilities and expertise available, and the presence and severity of comorbid conditions.

In this respect, the American College of Cardiology, American Heart Association, and European Society of Cardiology 2006 guidelines have done an admirable job in addressing changing concepts with regard to the pathogenesis and classification of atrial fibrillation, and the diverse scope of the therapeutic armamentarium relating to different categories of patients. One of the strengths of the guidelines is that they grade levels of evidence, which enables one to put the current body of information in perspective. Since the first release of the guidelines in 2001, a steadily growing amount of evidence has been published, both positive and negative, from the large trials of rhythm versus rate control and prevention of thromboembolism, and from the smaller trials and registry studies of catheter-based and surgical approaches to the maintenance of sinus rhythm.

Atrial fibrillation is one of the three growing epidemics of cardiovascular disease. The disturbing increase in incidence and prevalence of atrial fibrillation is in part a function

\section{Atrial \\ fibrillation \\ remains the \\ most common \\ clinical \\ arrhythmia \\ and new \\ approaches to \\ prevention and \\ treatment are \\ needed.}

$B J$ Gersh is an

Advisory Board

member for Nature

Clinical Practice

Cardiovascular

Medicine and

$D$ Packer is Professor

of Medicine at

the Division of

Cardiovascular

Diseases, Mayo Clinic, and Mayo Clinic College of Medicine, Rochester, MN, USA.

\section{Competing interests}

The authors declared

they have no competing

interests.

www.nature.com/clinicalpractice doi:10.1038/ncpcardio0796 of the aging of the population, but other factors seem to have an important role and are currently the focus of intense investigation. Atrial fibrillation remains the most common clinical arrhythmia and new approaches to prevention and treatment are needed.

When the guidelines are next updated, many of the gaps that currently exist in the context of evidence-based medicine will hopefully be closed. Large ongoing trials are addressing multiple issues including advances in catheter-based ablation technologies, alternative methods of anticoagulation, the pressing need for novel antiarrhythmic drugs, and the role of 'upstream' therapies in the prevention of atrial fibrillation (e.g. antagonists of the reninangiotensin-aldosterone system, statins and fish oils). The controversy that surrounds rate versus rhythm control in older, at-risk patients who are mildly symptomatic has been largely settled by the trials of the 1990s. Nonetheless, we do not yet have all the answers, particularly for patients with congestive heart failure, noncompliant left ventricular disorders such as hypertrophic cardiomyopathy and 'diastolic' heart failure, younger patients, and those in whom arrhythmia is poorly tolerated. In patients treated primarily with rate control, an unresolved question currently being addressed is whether lenient versus strict rate control have different effects on morbidity, mortality, and quality of life.

The guideline process continuously evolves, and the committee members of the American College of Cardiology, the American Heart Association, and the European Society of Cardiology 2006 guidelines should be congratulated for their synthesis of an enormous amount of information. All we can say for certain with regard to the next generation of guidelines is that there will be changes, and that these will be evidence-based. 